\title{
MiR-449b-5p targets IncRNA PSMG3-AS1 to suppress cancer cell proliferation in lung adenocarcinoma
}

\author{
Na Yue, Ming Ye, Ran Zhang and Yunquan Guo*
}

\begin{abstract}
Background: PSMG3-AS1 has been characterized as an oncogenic IncRNA in breast cancer, while its role in other cancers is unknown. This study investigated the role of PSMG3-AS1 in lung adenocarcinoma (LUAD).

Methods: This study included 64 LUAD patients (42 males and 22 females) who were enrolled between May 2012 and May 2014. RT-qPCR was used to evaluate the expression levels of IncRNA. Cell proliferation analysis was performed using CCK-8 kit.

Results: We found that upregulation of PSMG3-AS1 in LUAD predicted the poor survival of patients. MiR-449b-5p is downregulated in LUAD and the expression levels of LUAD were inversely correlated with the expression levels of PSMG3-AS1. MiR-449b-5p was predicted to target PSMG3-AS1, and overexpression of miR-449b-5p resulted in the downregulation of PSMG3-AS1 in LUAD cells. Cell proliferation analysis showed that overexpression of PSMG3-AS1 resulted in increased rate of cell proliferation. Overexpression of miR-449b-5p reduced the enhancing effects of PSMG3-AS1 on cell proliferation.
\end{abstract}

Conclusions: Therefore, miR-449b-5p may target PSMG3-AS1 in LUAD to suppress cancer cell proliferation.

Keywords: Lung adenocarcinoma, PSMG3-AS1, miR-449b-5p, Prognosis

\section{Background}

Lung cancer is considered as the leading cause of cancer-related mortality for decades worldwide [1]. The latest GLOBOCAN statistics reported that lung cancer caused 1,761,007 deaths, accounting for $18.4 \%$ of all cancer deaths in 2018 [2]. In the same year, there were 2, 093,876 new cases of lung cancer, accounting for $11.6 \%$ of all new cancer cases [2]. It is estimated that more than $50 \%$ of lung cancer patients diagnosed with a localized tumor can live longer than 5 years, but only $16 \%$ of lung cancer patients are diagnosed at early stages [3]. Once distant metastasis occurs, only $6 \%$ of lung cancer

\footnotetext{
* Correspondence: rq8980@163.com

Department of Pathology, the 3rd Affiliated Teaching Hospital of Xinjiang Medical University (Affiliated Cancer Hospital), NO.789 East Suzhou Street, New Urban Area, Urumqi 830000, Xinjiang, China
}

patients can survive for 5 years [4]. Tobacco smoking is responsible for the majority of lung cancer cases, while this disease also affects never-smokers, indicating the complicated pathogenesis $[5,6]$. Studies on the molecular mechanisms of lung cancer have identified critical molecular pathways involved in the development and progression of this disease $[7,8]$. Characterization of lncRNAs involved in lung cancer provides novel insights into the development of targeted therapies $[9,10]$. Noncoding RNAs (ncRNAs), such as long (> $200 \mathrm{nt}$ ) ncRNAs (lncRNAs) [11] and miRNAs [12] are critical players in cancer biology and either promote or suppress cancer development by regulating the expression of cancerrelated genes. Therefore, regulating the expression of cancer-related ncRNAs may benefit cancer treatment. However, the functions of most ncRNAs in cancer

C C The Author(s). 2020 Open Access This article is licensed under a Creative Commons Attribution 4.0 International License, which permits use, sharing, adaptation, distribution and reproduction in any medium or format, as long as you give appropriate credit to the original author(s) and the source, provide a link to the Creative Commons licence, and indicate if changes were made. The images or other third party material in this article are included in the article's Creative Commons licence, unless indicated otherwise in a credit line to the material. If material is not included in the article's Creative Commons licence and your intended use is not permitted by statutory regulation or exceeds the permitted use, you will need to obtain permission directly from the copyright holder. To view a copy of this licence, visit http://creativecommons.org/licenses/by/4.0/ The Creative Commons Public Domain Dedication waiver (http://creativecommons.org/publicdomain/zero/1.0/) applies to the data made available in this article, unless otherwise stated in a credit line to the data. 
remain unclear. PSMG3-AS1 was recently characterized as an oncogenic lncRNA in breast cancer [13], while its role in lung cancer remains unclear. Our bioinformatics analysis showed that PSMG3-AS1 may be targeted by miR-449b-5p, which is a tumor suppressive miRNA [14]. This study was therefore carried out to investigate the interaction between miR-449b-5p and PSMG3-AS1 in lung adenocarcinoma, a major subtype of lung cancer.

\section{Methods}

\section{LUAD patients and tissue samples}

This study was approved by the Ethics Committee of the 3rd Affiliated Teaching Hospital of Xinjiang Medical University. This study included 64 LUAD patients (42 males and 22 females) who were enrolled at the aforementioned hospital between May 2012 and May 2014. All LUAD patients were newly diagnosed cases and cases with other severe clinical disorders, such as other malignancies and chronic diseases, were excluded. No therapy was initiated before this study. The age of patients ranged from 42 to 66 years old, with a mean age of $54.2 \pm 6.7$ years old. Among the 64 patients, 51 were smokers or had a history of smoking. All patients signed the written informed consent. Paired LUAD and nontumor tissues were collected from each patient through fine needle aspiration (FNA). All tissue specimens were confirmed by histopathological exams. All tissue samples were stored in liquid nitrogen before use.

\section{A 5-year follow-up}

The 64 patients were staged according to AJCC criteria (8th edition). There were $8,11,21$, and 24 cases of stage I, stage II, stage III and stage IV, respectively. Based on clinical stage, patients were either treated with chemotherapy, surgical resection, radiotherapy, targeted therapy, or the combination of these treatments. All patients were followed up for 5 years after admission to record their survival. All patients completed the follow-up.

\section{LUAD cells and transfections}

LUAD cell lines H522 and H23 cells obtained from ATCC were used. Cell culture medium was composed of $10 \%$ FBS and 90\% RPMI-1640 medium. Cell culture conditions were $5 \% \mathrm{CO}_{2}, 37^{\circ} \mathrm{C}$ and $95 \%$ humidity. PSMG3-AS1 expression vector was constructed using pcDNA3.1 vector (Sigma-Aldrich) as backbone. Negative control (NC) miRNA (5'-CCGUAGGUGCACGUGAAACGAC-3') and miR-449b-5p mimic (5'-AGGCAGUGUAUUGUUAGC UGGC-3') were also purchased from Sigma-Aldrich. PSMG3-AS1 siRNA (5'-CUGUGCGUUCGUUCUGCU UC-3') and NC siRNA (5'-GUCGUAGUUACCUU UGACGAU-3') were purchased from Invitrogen. H522 and $\mathrm{H} 23$ cells were harvested at about $85 \%$ confluence. Lipofectamine 2000 (Invitrogen) was used to transfect the cells with $45 \mathrm{nM}$ siRNA or $10 \mathrm{nM}$ expression vector. Untransfected cells were used as the Control (C) cells, and NC miRNA- or empty vector-infected cells were used as $\mathrm{NC}$ cells. Cells were harvested $48 \mathrm{~h}$ later to perform the subsequent experiments.

\section{Prediction of the interaction between miR-449b-5p and PSMG3-AS1}

IntaRNA 2.0 (http://rna.informatik.uni-freiburg.de/IntaRNA/ Input.jsp) was used to predict the interaction between miR449b-5p and PSMG3-AS1. The sequence of miR-449b-5p was input as short sequence and PSMG3-AS1 sequence was long sequence. All other parameters were set as default.

\section{RNA preparations}

RNAzol (Sigma-Aldrich) was used to isolate total RNA from tissue samples and in vitro cultivated cells. To harvest miRNAs, RNA precipitation and washing were performed using $85 \%$ ethanol. The gDNA eraser (Takara Bio) was used to remove genomic DNA from all RNA samples. NanoDrop 2000 Spectrophotometer (Thermo Scientific) was used to measure RNA concentrations. RNA integrity was checked by Urea-PAGE gel.
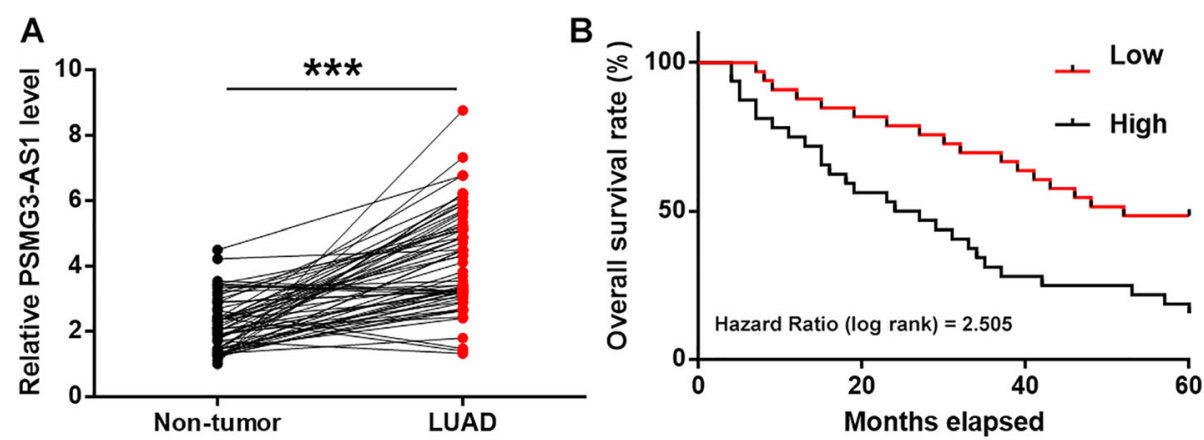

Fig. 1 Upregulation of PSMG3-AS1 in LUAD predicted poor survival. Expression levels of PSMG3-AS1 in both LUAD and non-tumor tissues from 64 patients with LUAD were measured by performing RT-qPCR. PCR reactions were repeated 3 times and mean values were presented and compare (a). ${ }^{* *}, p<0.001$. The 64 patients were divided into high and low PSMG3-AS1 level groups $(n=32)$ with the mean value of PSMG3-AS1 expression level in LUAD tissues as a cutoff value. Survival curves were plotted and compared by log-rank test (b) 
Table 1 Chi-squared test analysis of the correlations between expression levels of PSMG3-AS1 and patients' clinical data

\begin{tabular}{|c|c|c|c|c|c|c|}
\hline Items & Groups & Cases & High-expression $(n=32)$ & Low-expression $(n=32)$ & $x^{2}$ & $p$ value \\
\hline \multirow[t]{2}{*}{ Age (years) } & $>=55$ & 33 & 14 & 19 & 1.56 & 0.21 \\
\hline & $<55$ & 31 & 18 & 13 & & \\
\hline \multirow[t]{2}{*}{ Gender } & Male & 42 & 22 & 20 & 0.28 & 0.60 \\
\hline & Female & 22 & 10 & 12 & & \\
\hline \multirow[t]{4}{*}{ AJCC } & । & 8 & 4 & 4 & 1.19 & 0.76 \\
\hline & $\|$ & 11 & 6 & 5 & & \\
\hline & III & 21 & 12 & 9 & & \\
\hline & IV & 24 & 10 & 14 & & \\
\hline \multirow[t]{2}{*}{ Smoking } & Yes & 51 & 24 & 27 & 0.87 & 0.35 \\
\hline & No & 13 & 8 & 5 & & \\
\hline
\end{tabular}

\section{RT-qPCR}

Precision nanoScript2 Reverse Transcription Kit (Primerdesign) was used to reverse transcribe total RNA samples into cDNA. With cDNA as template, qPCR reactions were prepared using SingleShot ${ }^{\mathrm{TM}} \mathrm{SYBR}^{\circ}$ Green Kit (Bio-Rad). The expression levels of PSMG3-AS1 were measured with GAPDH as endogenous control. To measure the expression levels of mature miR-449b-5p, All-in-One miRNA qRT-PCR Detection Kit (GeneCopoeia) was used to complete all steps including polyadenylation, reverse transcription and qPCR assays. U6 was used as the endogenous control of miR-449b-5p. PCR reactions were repeated 3 times and $2^{-\Delta \Delta \mathrm{Ct}}$ method was used to normalize gene expression levels. Primer sequences used were as following: 5' -GAAGCAGAACCAACGCACAG-3' (forward) and 5' GCATAATCCAATCCCTCAAGAA-3' (reverse) for PSMG3-AS1; 5'-GTCTCCTCTGACTTCAACAGCG-3' (forward) and 5'-ACCACCCTGTTGCTGTAGCCAA-3' (reverse) for GAPDH. Forward primer of miR-449b-5p was 5' -AGGCAGTGTATTGTTAGCT-3'. U6 forward primer and the universal reverse primer were from the kit.

\section{Cell counting Kit-8 (CCK-8) assay}

$\mathrm{H} 522$ and $\mathrm{H} 23$ cells harvested at $48 \mathrm{~h}$ post-transfection were subjected to cell proliferation analysis using a CCK-8 kit (Dojindo). Each well of a 96-well cell culture plate was filled with 5000 cells in $0.1 \mathrm{ml}$ medium. Cells were cultivated under aforementioned conditions and OD values were measured every $24 \mathrm{~h}$ for a total of $4 \mathrm{~d}$. CCK-8 solution was added into each well at $4 \mathrm{~h}$ before the measurement of OD values.

\section{Statistical analysis}

Mean \pm SEM values were used to express data from 3 independent replicates. All statistical analyses were performed using GraphPad Prism6 software (GraphPad, USA). The comparisons between non-tumor and LUAD tissues were performed by paired $t$ test. Comparisons among multiple groups were performed by ANOVA (one-way) and Tukey test. Linear regression was used for correlation analysis. The 64 patients were divided into high $(n=32)$ and low $(n=32)$ PSMG3-AS1 level groups with the mean value of PSMG3-AS1 expression
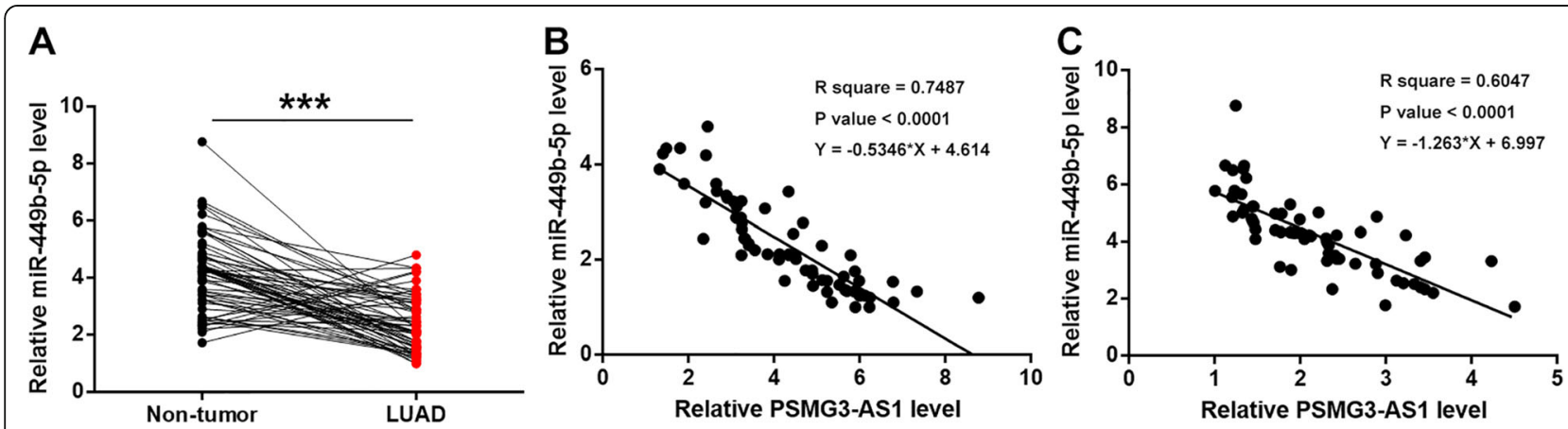

Fig. 2 MiR-449b-5p is downregulated in LUAD and inversely correlated with PSMG3-AS1. Expression levels of miR-449b-5p in both LUAD and non-tumor tissues from the 64 patients with LUAD were also measured by performing RT-qPCR. PCR reactions were repeated 3 times and mean values were presented and compare $(\mathbf{a}) .{ }^{* * *}, p<0.001$. Correlations between expression levels of miR-449b-5p and PSMG3-AS1 across both LUAD (b) and non-tumor (c) tissues were analyzed by linear regression 
A
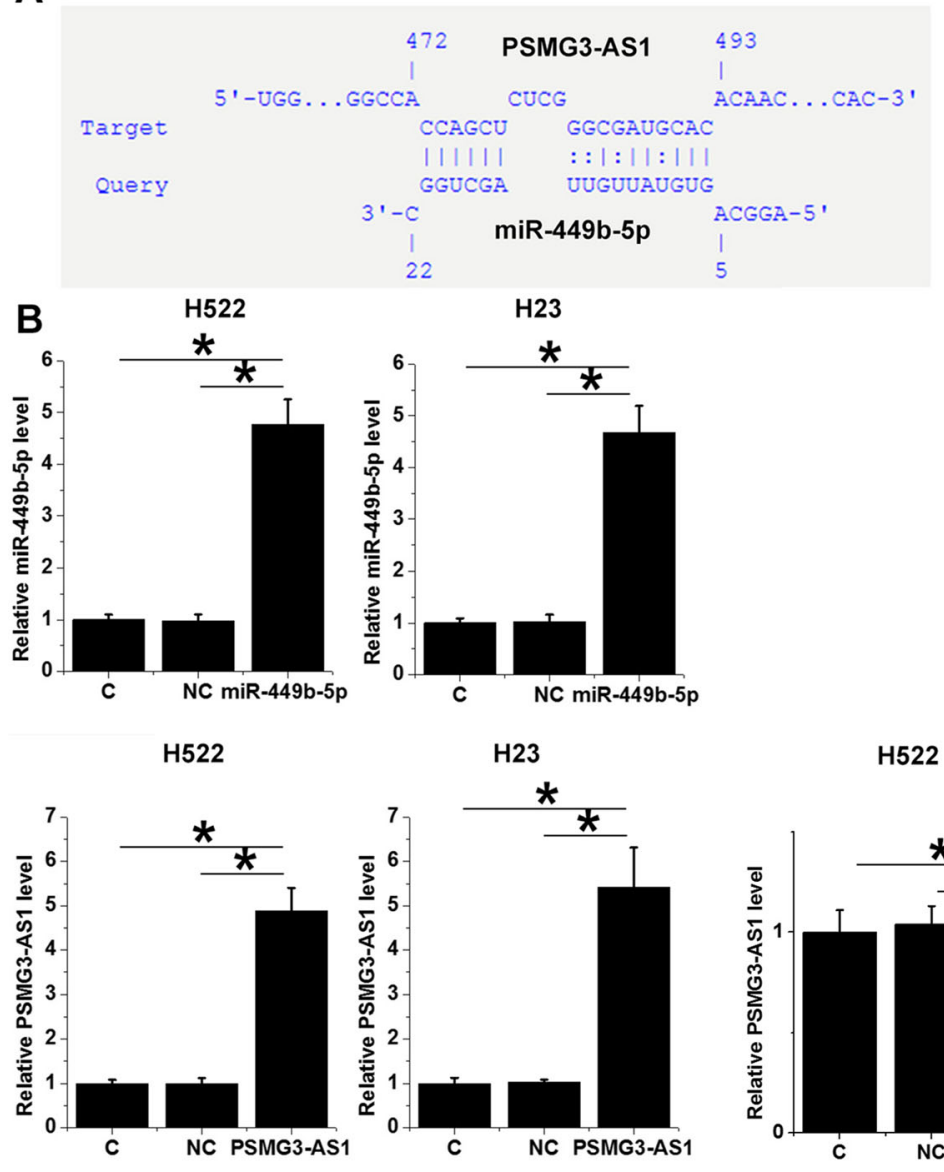

H522

H23

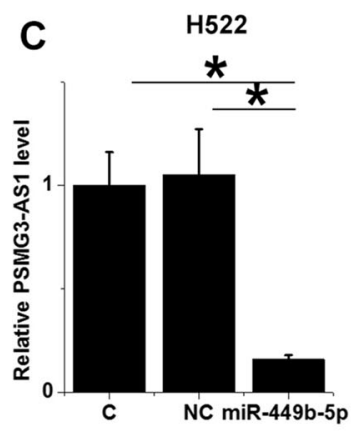

H23
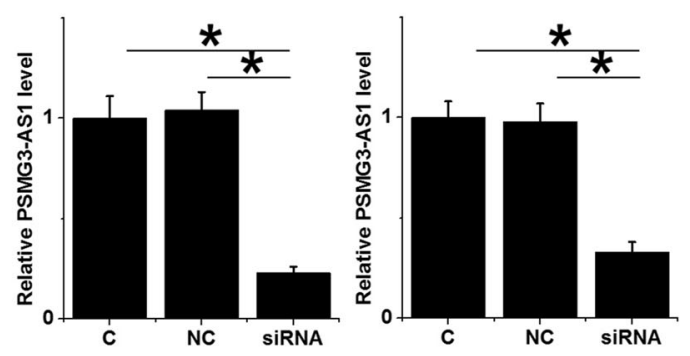

D

H522
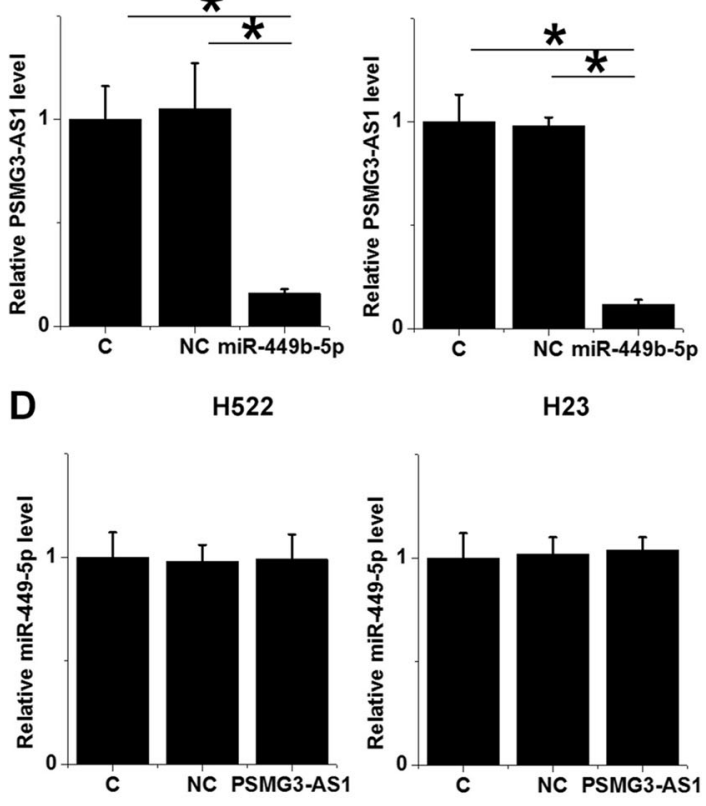

$\mathrm{H} 23$

H522

H23
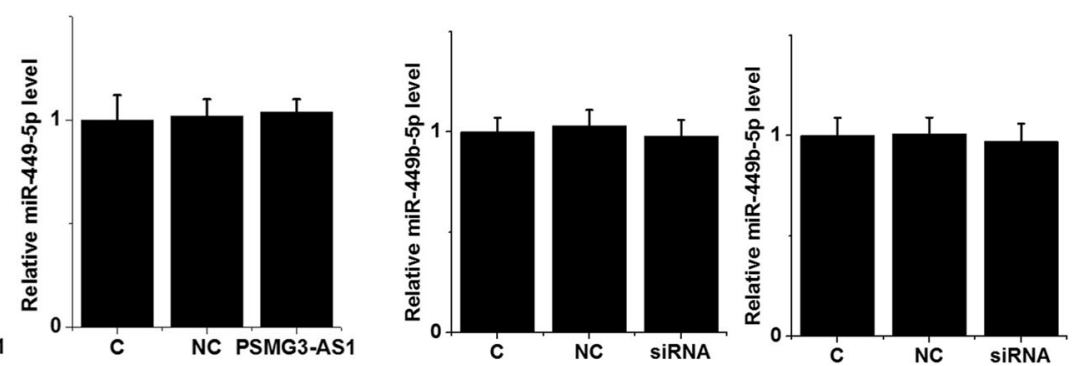

Fig. 3 (See legend on next page.) 
(See figure on previous page.)

Fig. 3 MiR-449b-5p targeted PSMG3-AS1 to downregulate its expression. The interaction between miR-449b-5p and PSMG3-AS1 was predicted using IntaRNA 2.0 (a). H522 and H23 cells were transfected with miR-449b-5p mimic, PSMG3-AS1 expression vector, or PSMG3-AS1, followed by the confirmation of miR-449b-5p and PSMG3-AS1 overexpression as well as PSMG3-AS1 siRNA silencing by RT-qPCR at $48 \mathrm{~h}$ post-transfection (b). The effects of overexpression of miR-449b-5p on PSMG3-AS1 (c) and the effects of overexpression of and silencing of PSMG3-AS1 on miR-449b-5p (d) were also analyzed by RT-qPCR at $48 \mathrm{~h}$ post-transfection. All experiments were repeated 3 times and mean values were presented and compared. *, $p<0.05$

level in LUAD tissues used as a cutoff value. Survival curves were plotted based on the 5-year follow-up data and compared by log-rank test. Chi-squared test was performed to analyze the relationship between the expression levels of PSMG3-AS1 and patients' clinical data. $p<0.05$ was considered as statistically significant.

\section{Results}

Upregulation of PSMG3-AS1 in LUAD predicted poor survival The expression levels of PSMG3-AS1 in both LUAD and non-tumor tissues from the 64 patients with LUAD were measured by RT-qPCR. Compared to non-tumor tissues, the expression levels of PSMG3-AS1 were significantly higher in LUAD tissues (Fig. 1a, $p<0.05$ ). Survival curves for both high and low PSMG3-AS1 level groups were plotted. No significant differences in therapeutic treatments were found between high and low PSMG3-AS1 level groups. Compared to the low PSMG3-AS1 level group, patients in the high PSMG3-AS1 level group showed higher mortality rate within 5-year follow-up. Chi-squared test analysis showed that the expression levels of PSMG3-AS1 were not significantly correlated with patients' age, gender, clinical stages and smoking habit (Table 1).

\section{MiR-449b-5p is downregulated in LUAD and inversely correlated with PSMG3-AS1}

The expression levels of miR-449b-5p in both LUAD and non-tumor tissues from the 64 patients with LUAD were also measured by RT-qPCR. Compared to non-tumor tissues, the expression levels of miR-449b-5p were significantly lower in LUAD tissues (Fig. $2 \mathrm{a}, p<0.001$ ). Correlation analysis showed that the expression levels of miR-449b-5p and PSMG3-AS1 were inversely and significantly correlated across both LUAD (Fig. 2b) and non-tumor (Fig. 2c) tissues.

\section{MiR-449b-5p targeted PSMG3-AS1 to downregulate its expression}

The interaction between miR-449b-5p and PSMG3-AS1 was predicted using IntaRNA 2.0 [15]. It was observed that miR-449b-5p and PSMG3-AS1 could form multiple bases pairing (Fig. 3a). H522 and H23 cells were transfected with miR-449b-5p mimic, PSMG3-AS1 expression vector, or PSMG3-AS1 siRNA. Overexpression of miR-449b-5p and PSMG3-AS1, and the silencing of PSMG3-AS1 were confirmed by RT-qPCR at $48 \mathrm{~h}$ posttransfection (Fig. $3 \mathrm{~b}, p<0.05$ ). Compared to the $\mathrm{C}$ and $\mathrm{NC}$ groups, overexpression of miR-449b-5p resulted in downregulation of PSMG3-AS1 in cells of both cell lines (Fig. 3c, $p<0.05$ ). In contrast, overexpression of and silencing of PSMG3-AS1 did not affect the expression of miR-449b-5p (Fig. 3d).

\section{MiR-449b-5p targeted PSMG3-AS1 to suppress cancer cell proliferation}

The effects of overexpression of miR-449b-5p and PSMG3-AS1 on the proliferation of $\mathrm{H} 522$ and $\mathrm{H} 23$ cells were analyzed by performing a CCK-8 assay. Compared to the C group, overexpression of PSMG3-AS1 resulted
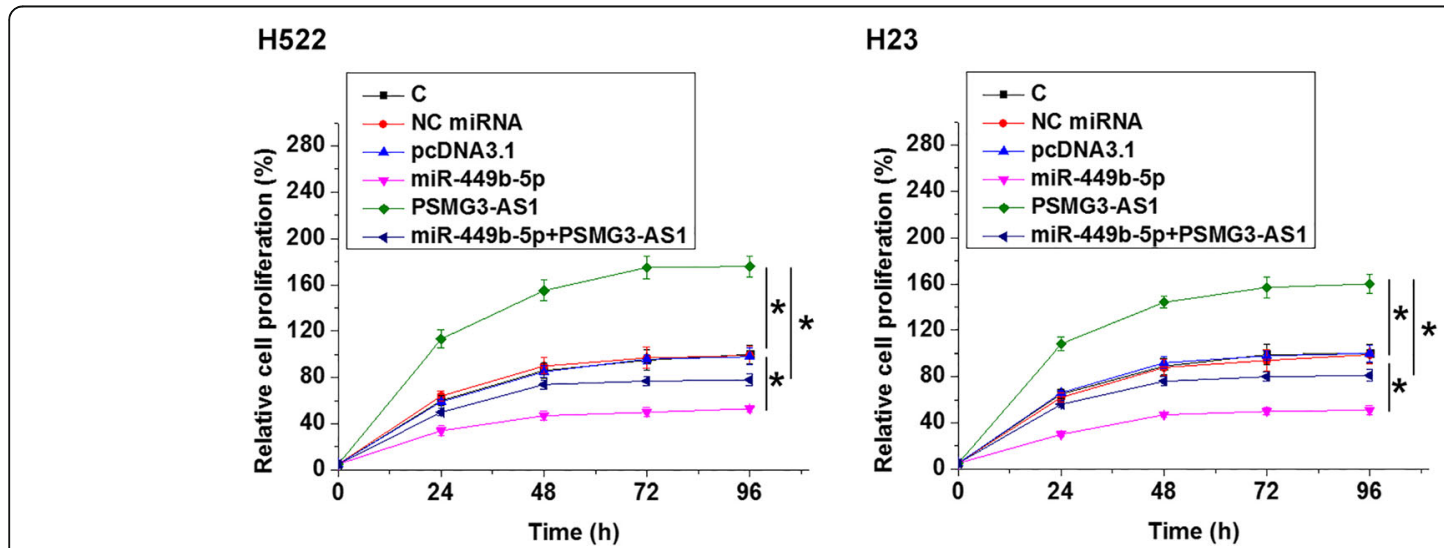

Fig. 4 MiR-449b-5p targeted PSMG3-AS1 to suppress cancer cell proliferation. The effects of overexpression of miR-449b-5p and PSMG3-AS1 on the proliferation of $\mathrm{H} 522$ and $\mathrm{H} 23$ cells were analyzed by performing CCK-8 assay. All experiments were repeated 3 times and mean values were presented and compared. *,$p<0.05$ 


\section{H522}

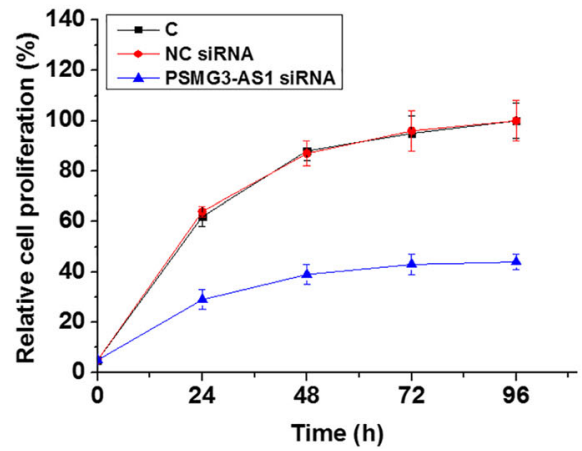

H23

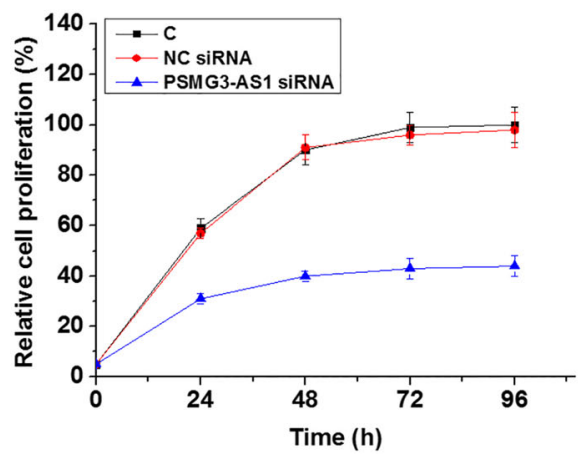

Fig. 5 PSMG3-AS1 siRNA silencing suppressed the proliferation of LUAD cells. The effects of silencing of PSMG3-AS1 on the proliferation of H522 and $\mathrm{H} 23$ cells were analyzed by performing CCK-8 assay. All experiments were repeated 3 times and mean values were presented and compared. *, $p<0.05$

in an increased rate of cell proliferation. Overexpression of miR-449b-5p played an opposite role and reduced the enhancing effects of PSMG3-AS1 on cell proliferation (Fig. 4, $p<0.05$ ). In addition, silencing of PSMG3-AS1 was also performed to further confirm the function of PSMG3-AS1 in regulating cell proliferation. Compared to the $\mathrm{C}$ group, silencing of PSMG3-AS1 resulted in a decreased proliferation of $\mathrm{H} 522$ and $\mathrm{H} 23$ cells (Fig. 5, $p<0.05)$.

\section{Discussion}

This study mainly investigated the interaction between miR-449b-5p and PSMG3-AS1 in LUAD. We found that the expression of miR-449b-5p and PSMG3-AS1 were altered in LUAD. In addition, miR-449b-5p might be able to target PSMG3-AS1 to suppress cancer cell proliferation.

The functionality of PSMG3-AS1 has only been investigated in breast cancer [13]. It is observed that PSMG3AS1 is overexpressed in breast cancer and may promote the migration and proliferation of cancer cells by sponging miR-143-3p [13]. To the best of our knowledge, this study is the first to report the upregulation of PSMG3AS1 in LUAD, a major subtype of lung cancer. Our in vitro cell experiments also showed that PSMG3-AS1 could promote the proliferation of LUAD cells. Therefore, our data suggested that PSMG3-AS1 played an oncogenic role in LUAD.

Based on our knowledge, the prognostic value of PSMG3-AS1 in cancers remains unknown. Our 5-year follow-up study showed that high expression levels of PSMG3-AS1 measured before therapy were closely correlated with the poor survival of LUAD patients. Most lung cancer patients are diagnosed at advanced stages and the survival is generally poor [16]. Due the lack of early diagnostic markers, the low early diagnostic rate of lung cancer is unlikely to be increased in the near future.
Therefore, as an alternative approach, accurate prognosis might help to determine therapeutic approaches and the development of personalized care program, thereby improving the overall survival.

MiR-449b-5p has been characterized as a tumor suppressive miRNA in multiple cancers, such as breast cancer and osteosarcoma $[14,17]$. In these cancers, miR-449b-5p targets multiple protein-coding genes, such as CREPT and c-Met, to suppress cancer development and progression $[14,17]$. This study is the first to show the downregulation of miR-449b-5p in LUAD and its inhibitory effects on LUAD cell proliferation. Therefore, miR-449b-5p is also a tumor suppressive miRNA in LUAD. Interesting, we proved that miR-449b-5p could target PSMG3-AS1. Therefore, besides protein-coding genes, miR-449b-5p can also target lncRNA to participate in cancer biology.

\section{Conclusions}

In conclusion, miR-449b-5p is downregulated in LUAD and PSMG3-AS1 is upregulated in LUAD. In addition, miR-449b-5p can target PSMG3-AS1 to suppress cancer cell proliferation.

\section{Abbreviations \\ LUAD: Lung adenocarcinoma; IncRNAs: Long non-coding RNAs; ncRNAs: Non-coding RNAs}

\section{Acknowledgments}

Not applicable.

\section{Authors' contributions}

NY: manuscript writing, literature search, and data analysis; MY and RZ: clinical research, data analysis and statistical analysis. YQG: manuscript writing, literature search and research design. All authors read and approved the final manuscript

Funding

Not applicable.

\section{Availability of data and materials}

The datasets used and/or analyzed during the current study are available from the corresponding author on reasonable request. 


\section{Ethics approval and consent to participate}

This study was approved by the Ethics Committee of the 3rd Affiliated Teaching Hospital of Xinjiang Medical University. All procedures performed in studies involving human participants were in accordance with the ethical standards of the institutional and national research committee. Written informed consent was obtained from all individual participants included in the study.

\section{Consent for publication}

Not applicable.

\section{Competing interests}

The authors declare that they have no competing interests.

Received: 18 December 2019 Accepted: 18 May 2020

Published online: 29 May 2020

\section{References}

1. Siegel RL, Miller KD, Jemal A. Cancer statistics, 2019. CA Cancer J Clin. 2019; 69(1):7-34.

2. Bray F, Ferlay J, Soerjomataram I, Siegel RL, Torre LA, Jemal A. Global cancer statistics 2018: GLOBOCAN estimates of incidence and mortality worldwide for 36 cancers in 185 countries. CA Cancer J Clin. 2018;68(6):394-424.

3. Inage T, Nakajima T, Yoshino I, Yasufuku K. Early lung Cancer detection. Clin Chest Med. 2018;39(1):45-55.

4. Luo YH, Luo L, Wampfler JA, Wang Y, Liu D, Chen YM, et al. 5-year overall survival in patients with lung cancer eligible or ineligible for screening according to US preventive services task force criteria: a prospective, observational cohort study. Lancet Oncol. 2019;20(8):1098-108.

5. Korpanty GJ, Kamel-Reid S, Pintilie M, Hwang DM, Zer A, Liu G, et al. Lung cancer in never smokers from the Princess Margaret Cancer Centre. Oncotarget. 2018;9(32):22559-70.

6. Subramanian J, Govindan R. Lung cancer in never smokers: a review. J Clin Oncol. 2007;25(5):561-70.

7. Borczuk AC, Gorenstein L, Walter KL, Assaad AA, Wang L, Powell CA. Nonsmall-cell lung cancer molecular signatures recapitulate lung developmental pathways. Am J Pathol. 2003;163(5):1949-60

8. Cooper WA, Lam DC, O'Toole SA, Minna JD. Molecular biology of lung cancer. J Thorac Dis. 2013;5(Suppl 5):S479-90.

9. Chan BA, Hughes BG. Targeted therapy for non-small cell lung cancer: current standards and the promise of the future. Transl Lung Cancer Res. 2015;4(1):36-54

10. Yao Z, Fenoglio S, Gao DC, Camiolo M, Stiles B, Lindsted T, et al. TGF-beta IL- 6 axis mediates selective and adaptive mechanisms of resistance to molecular targeted therapy in lung cancer. Proc Natl Acad Sci U S A. 2010 107(35):15535-40.

11. Tano K, Akimitsu N. Long non-coding RNAs in cancer progression. Front Genet. 2012;3:219.

12. Farazi TA, Spitzer Jl, Morozov P, Tuschl T. miRNAs in human cancer. J Pathol. 2011;223(2):102-15.

13. Cui Y, Fan Y, Zhao G, Zhang Q, Bao Y, Cui Y, et al. Novel IncRNA PSMG3AS1 functions as a miR-143-3p sponge to increase the proliferation and migration of breast cancer cells. Oncol Rep. 2019;43(1):229-39.

14. Jiang J, Yang X, He X, Ma W, Wang J, Zhou Q, et al. MicroRNA-449b-5p suppresses the growth and invasion of breast cancer cells via inhibiting CREPT-mediated Wnt/beta-catenin signaling. Chem Biol Interact. 2019; 302:74-82.

15. Mann M, Wright PR, Backofen R. IntaRNA 2.0: enhanced and customizable prediction of RNA-RNA interactions. Nucleic Acids Res. 2017;45(W1):W435-W9.

16. Sperduto PW, Yang TJ, Beal K, Pan H, Brown PD, Bangdiwala A, et al. Estimating survival in patients with lung Cancer and brain metastases: an update of the graded prognostic assessment for lung Cancer using molecular markers (lung-molGPA). JAMA Oncol. 2017;3(6):827-31.

17. Li Q, Lu C, Wang J, Gao M, Gao W. MicroRNA-449b-5p suppresses proliferation, migration, and invasion of osteosarcoma by targeting c-met. Med Sci Monit. 2019;25:6236-43.

\section{Publisher's Note}

Springer Nature remains neutral with regard to jurisdictional claims in published maps and institutional affiliations.

Ready to submit your research? Choose BMC and benefit from:

- fast, convenient online submission

- thorough peer review by experienced researchers in your field

- rapid publication on acceptance

- support for research data, including large and complex data types

- gold Open Access which fosters wider collaboration and increased citations

- maximum visibility for your research: over $100 \mathrm{M}$ website views per year

At BMC, research is always in progress.

Learn more biomedcentral.com/submissions 